\title{
Dry powder cationic lipopolymeric nanomicelle inhalation for targeted delivery of antitubercular drug to alveolar macrophage
}

This article was published in the following Dove Press journal:

International Journal of Nanomedicine

10 August 2013

Number of times this article has been viewed

\author{
Mithun Varghese Vadakkan' \\ K Annapoorna ${ }^{2}$ \\ KC Sivakumar ${ }^{3}$ \\ Sathish Mundayoor ${ }^{2}$ \\ GS Vinod Kumar' \\ 'Chemical Biology, ${ }^{2}$ Mycobacterium \\ Research Group, ${ }^{3}$ Bioinformatics \\ Facility, Rajiv Gandhi Centre for \\ Biotechnology, Thiruvananthapuram, \\ Kerala, India
}

\begin{abstract}
Excipients having self-assembling properties are less explored in the field of dry powder inhalation (DPI) technology. An amphiphilic lipopolymer system was developed using stearic acid (SA) and branched polyethyleneimine (BPEI) (1800 Dalton), at different proportions by covalent conjugation. A molecular dynamic (MD) simulation tool was employed for predicting the carrier behavior in a polar in vivo condition. The structural characterization was carried out using nuclear magnetic resonance spectroscopy (NMR) and Fourier transform infrared (FTIR) spectroscopy. The physical nature of the lipopolymer was analyzed by differential scanning calorimetry. Determination of zeta potential and diameter of the micelles showed existence of cationic particles in the nano size range when a lower number of primary amino groups of BPEI was grafted with SA. The rifampicin (RIF)-loaded lipopolymer was also formulated further into spray-dried microparticles. Powder X-ray diffraction (PXRD) studies revealed that the RIF API (active pharmaceutical ingredient) exists as molecular dispersion in spray-dried microparticles. Topological analysis of the spray-dried nanomicelle was carried out using scanning electron microscopy (SEM). A large population of the drug-carrying particles were found to be under the inhalable size range (fine particle fraction $67.88 \% \pm 3 \%$ ). In vitro drug release kinetics from spray-dried nanomicelles were carried out at lung fluid $\mathrm{pH}$.
\end{abstract}

Keywords: molecular dynamics, dry powder inhalation, pulmonary tuberculosis, nanomicelle, lipopolymer, rifampicin

\section{Introduction}

After approval of the first inhalable antimicrobial agent $\left(\mathrm{Nebupent}^{\mathbb{B}}\right.$; Fresenius SE and Co, KGaA, Bad Homburg, Germany) by the US Food and Drug Administration (FDA), researchers realized the potentials of the inhalation route, especially for treating ailments affecting the lungs. Compared to conventional oral therapy, inhalation drug delivery proved to be superior in delivering high payloads of drug to deep in the lung (alveoli). ${ }^{1}$ The limited clearance of deposited materials from the lung is the greatest deterrent in developing inhalable formulations. Pressurized inhalers were the first generation of portable inhalation dosage forms. They need patient coordination and the loss of drug in the oropharyngeal region due to impaction is greater than when using dry powder inhalers (DPI). ${ }^{2}$ In recent years the importance of pressurized inhalers has waned due to environmental problems and the huge loss of the administered dose. ${ }^{3}$

It is noteworthy that particle engineering contributed many innovations in the field of DPI technology, including as microparticles, large porous particles, and nanofibers for the delivery of xenobiotics. ${ }^{1,4-7}$ Hickey and his coworkers reported first DPI formulation
Correspondence: GS Vinod Kumar Rajiv Gandhi Centre for Biotechnology, Thycaud PO, Thiruvananthapuram, 695014 Kerala, India Tel $+9 \mid$ 47| 2529526 Fax $+9 \mid$ 47। 2348096 Email gsvinod@rgcb.res.in 
for pulmonary tuberculosis using poly (lactic-co-glycolic acid) microspheres as carriers. ${ }^{8,9}$ In vivo study results on biodegradable microparticles containing antitubercular agents indicate better efficacy of these particles in controlling pulmonary tuberculosis when compared to the oral delivery route. ${ }^{10}$ However, it was observed by De and Robinson that poly (lactic-co-glycolic acid) (PLGA) microparticles undergo aggregation on storage and form solid bridges between the particles. $^{11}$

As the US FDA approved excipients for DPI application are very limited, active research is needed in this area. Polymeric nanoparticles have proven to be better in cargoing large amounts of drug. ${ }^{12,13}$ Self-assembling copolymers of both hydrophilic and hydrophobic moieties have been found to be more effective in delivering anticancer drugs to tumours. ${ }^{14-17}$ The self-assembling property of the excipient molecule fumaryl diketopiperazine has been explored as a platform technology (Technosphere ${ }^{\circledR}$; MannKind Corporation, Valencia, CA, USA) for delivery of both synthetic and biological molecules for targeting alveoli.

Apart from chemical incompatibilities, hydrophobicity of the drug or the excipients was the only consideration in preformulation studies for developing controlled drug delivery systems. Computational approaches are the new arena in rational designing of polymers for nanocarrier development. ${ }^{18}$ In addition to the gross property of carriers, individual atomic interactions between functional groups of a carrier and drug can also be predicted using computational approaches. ${ }^{19}$ Cationic polymers, especially low molecular weight (LMW) branched polyethyleneimine (BPEI) and their modified copolymers, are reported to be a safe vector for gene delivery applications. ${ }^{20-22}$ An amphiphilic unimolecular nanomicelle system was synthesized by tuning a hydrophobic and hydrophilic balance through grafting of stearic acid (SA) onto LMW BPEI by covalent conjugation in two different combinations, namely SABPEI 5050 and SABPEI 7030. Computational tools such as molecular dynamics (MD) and docking studies were used for studying intermicellar cohesional force of attraction, micellar stability, and drug-micelle interactions. In complement with the computational studies, wet lab experiments were also carried out for the evaluation of a synthesized lipopolymer as a DPI carrier.

The aim of the present work is to make an amphiphilic carrier system with cationic properties. The amphiphilic nature will help in deaggregation of the inhaled microparticles into nanomicelles. The cationic property is expected to give better cargoing of drug to the negatively charged cells.

\section{Materials and methods Materials}

SA, pyrene, trifluoroacetic acid (TFA), spectroscopy grade potassium bromide, Karl Fischer reagents, and all solvents were purchased from Merck Millipore (Billerica, MA, USA). BPEI 1800 Dalton (Alfa Aeser, Ward Hill, MA, USA) was used for the synthesis of both SABPEI 5050 and SABPEI 7030 combinations. $N, N^{\prime}$-Dicyclohexylcarbodiimide, $N$ hydroxysuccinimide, Roswell Park Memorial Institute (RPMI) 1640 media, Rhodamine B, and phosphate buffered saline (PBS) were procured from Sigma-Aldrich (St Louis, MO, USA). Rifampicin (RIF) IP was purchased from HiMedia Laboratories (Mumbai, India). Fetal bovine serum (FBS) was supplied by Life Technologies (Carlsbad, CA, USA). Inhalac 230 was provided by Meggle Pharma (Hamburg, Germany) as a gift sample.

\section{Synthesis and characterization of SABPEI combinations}

SA grafted BPEI lipopolymer was synthesized by forming a covalent amide linkage between the carboxyl group of SA with the amino functionality of BPEI, using $\mathrm{N}, \mathrm{N}$-Dicyclohexylcarbodiimide (DCC) as a dehydrating reagent. Briefly, SA was activated using a mixture of DCC and $N$-hydroxysuccinimide (NHS) in 0.5:1.2:1.2 molar ratios, respectively, using dichloromethane (DCM) as a solvent. The reaction was carried out in an inert nitrogen atmosphere, under stirring, at ambient temperature. After 24 hours the urea adduct formed was filtered out and polyethyleneimine previously dissolved in methanol was added to the activated SA. The above mixture was allowed to react for another 24 hours and the synthesized lipopolymers were purified by solubilization and reprecipitation in DCM/Diisopropyl ether. The lipopolymer was dispersed in double distilled water (MilliQ ${ }^{\circledR}$; Merck Millipore), then dialyzed against $10 \%$ ethanol solution using a dialysis membrane (Membracel dialysis tubing; molecular weight cut-off 3500 D; Serva Electrophoresis, Heidelberg, Germany) for 16 hours to remove byproducts and unreacted reagents. This was followed by dialysis against distilled water for 24 hours, with frequent replacement with fresh distilled water.

\section{Physicochemical characterization of the lipopolymer} The covalent linkage between SA and BPEI was confirmed by Fourier transform infrared (FTIR) spectroscopy and (nuclear magnetic resonance) NMR spectroscopy. FTIR 
spectroscopy was performed on a Spectrum 65 (PerkinElmer Inc, Waltham, MA, USA). Spectra were recorded between 4000 and $600 \mathrm{~cm}^{-1}$ wavenumber range. Dried samples were mixed with potassium bromide $(\mathrm{KBr})$ and further compressed into pellets for measurements. Proton NMR was recorded using a Bruker $500 \mathrm{MHz}$ spectrometer (Bruker Optik GmbH, Ettlingen, Germany).

Thermal properties such as $\mathrm{T}_{\mathrm{m}}$ (melting point temperature) and $\mathrm{T}_{\mathrm{g}}$ (Glass transition temperature) of the synthesized lipopolymer were determined by using differential scanning calorimetry (DSC) (DSC 6000, PerkinElmer Inc). Accurately weighed samples, sealed in an aluminum pan, were analyzed from $0{ }^{\circ} \mathrm{C}-100^{\circ} \mathrm{C}$ with a $10^{\circ} \mathrm{C} /$ minute heating rate under a nitrogen atmosphere.

The particle size and surface charge of different combinations of SABPEI were measured using a Zetasizer Nano ZS (Malvern Instruments, Malvern, UK) instrument at ambient temperature. The concentration of SABPEI suspension was $0.05 \mathrm{mg} / \mathrm{mL}$. Samples were analyzed without prior filtration.

\section{MD simulations and docking study}

The MD simulation was performed using GROMACS 4.5.4 simulation package, with the GROMACS96 force field on an Intel core i7 Processor workstation under CentOS 5.0. ${ }^{23}$ The topology files of SABPEIs, as needed in Gromacs, were prepared using the PRODRG server. ${ }^{24}$ The SABPEI 5050 molecule was solvated in a box containing the simple point charge (SPC) water model. The chosen dimension was large enough to include at least $8 \AA$ between the solutes and the edge of the box..$^{25}$ The total number of water molecules was 11,104 . Counter ions $\mathrm{Cl}^{-}$and $\mathrm{Na}^{+}$were added by replacing water molecules at random positions to achieve a neutral simulation box.

Prior to the MD simulations, internal constraints were relaxed by energy minimization. Following the minimization, an MD equilibration run was performed under position restraints for 100 picoseconds. During the MD runs, bond lengths were constrained using the Linear Constraint Solver (LINCS) algorithm and the water molecules were restrained using the SETTLE algorithm. ${ }^{26,27}$ Lennard-Jones and shortrange electrostatic interactions were calculated with 1.0 and $1.4 \mathrm{~nm}$ cutoffs, respectively. Long-range electrostatic forces were treated using the particle mesh Ewald method. ${ }^{28} \mathrm{~A}$ time step of 2 femtoseconds was used for the integration of equation of motion. The simulations were run under NPT (number of particles $[\mathrm{N}]$, system pressure $[\mathrm{P}]$, and temperature $[\mathrm{T}]$ ) conditions, using Berendsen's coupling algorithm for keeping the temperature and pressure constant $(\mathrm{P}=1$ bar, $\tau \mathrm{P}=1.0$ picoseconds; $\mathrm{T}=300 \mathrm{~K}, \tau \mathrm{T}=0.1$ picoseconds). ${ }^{29}$ Van der Waal forces were treated using a $12 \AA$ cutoff. The MD simulation was performed for 200 picoseconds and the coordinates were saved every 0.5 picosecond. The analysis of the simulations was performed using the analysis tools provided in the Gromacs package. Similar treatment was given to SABPEI 7030 during the simulation and docking studies.

\section{Determining biocompatibility}

Drug carriers are expected to release the drug inside the alveolar macrophages; thus, both the carrier and the drugloaded particles have to be safe for human use. It has been reported that Phorbol myristate acetate-differentiated human acute monocytic leukemia (THP-1) cells mimic the primary macrophages. ${ }^{30}$ The cytotoxicity of the SABPEI combinations and drug-loaded, spray-dried SABPEI were both evaluated using the THP-1 cell line. Cytotoxicity was determined following an earlier reported method, ie, the microculture tetrazolium method. ${ }^{31}$ Briefly, THP-1 cells were seeded to 96 well plates at a cell density of 5000 cells/well suspended in $200 \mu \mathrm{L}$ RPMI 1640 medium containing 10\% Fetal Bovine Serum (FBS) and $10 \mathrm{ng}$ of PMA. The plates were kept at $37^{\circ} \mathrm{C}$ for differentiation into macrophages. After 48 hours media were removed and cells were washed in PBS ( $\mathrm{pH} 7.4$ ). SABPEI, pure drug, and drug-loaded spray-dried SABPEI were added at five different concentrations after serial dilution in media. Each sample was analyzed in triplicate and after 24 hours of incubation in a $\mathrm{CO}_{2}$ chamber, the media were pipetted out and cells washed twice in PBS and $200 \mu \mathrm{L}$ of 3-(4,5-Dimethylthiazol-2-yl)-2,5-diphenyltetrazolium bromide (MTT) solubilized $(500 \mu \mathrm{g} / \mathrm{mL})$ in complete RPMI 1640 medium was added. After 4 hours of incubation, the supernatants were removed and formosan crystals present inside the living cell, were solubilized in $100 \mu \mathrm{L}$ isopropyl alcohol and cell viability was measured using a microplate reader (iMark; Bio-Rad Laboratories, Hercules, CA, USA) with a $570 \mathrm{~nm}$ filter.

\section{Cell uptake study}

THP-1 cells were differentiated on $25 \mathrm{~mm}$ round cover slips placed in $35 \mathrm{~mm}$ culture dishes. The differentiated cells were employed for the cell uptake study. The cells were infected with a green fluorescent protein (GFP)-tagged Mycobacterium smegmatis (M. smeg) with a multiplicity of infection (MOI) of 15. Cells along with the M. smeg were incubated further for 3 hours, for maximum internalization. The cells were washed with PBS after internalization 
and previously prepared rhodamine-entrapped SABPEI 5050 micelles dispersed in media were added. The above mentioned incubated cover slips were taken at predetermined time intervals ( 30 minutes, 1 hour, and 2 hours), washed, and fixed in paraformaldehyde solution. Prior to imaging, the cover slips were inverted and mounted on a glass slide applied with Fluoromount ${ }^{\circledR}$ (Sigma-Aldrich) as mounting medium. Images were captured using a laser confocal microscope (TCS SP2; Leica Microsystems, Wetzlar, Germany).

\section{Particle generation for inhalation}

The SABPEI 5050 (2.5 g) and RIF active pharmaceutical ingredient (API) $(2.5 \mathrm{~g})$ were dissolved in a mixture $(50 \mathrm{~mL})$ of DCM and methanol (80:20) at ambient temperature. The above prepared solution was spray-dried using Labultima LU 228 (Labultima, Mumbai, India) that had a two-fluid (cospray) pressure nozzle. Compressed nitrogen was used for atomization of the solution instead of air so as to create an inert atmosphere inside the spray-dryer. Inlet temperature was set to $40^{\circ} \mathrm{C}$ to achieve an outlet temperature of about $33^{\circ} \mathrm{C}$. The compressed nitrogen flow was maintained at $0.8 \mathrm{~kg} / \mathrm{cm}^{2}$ for optimum atomization and the aspiration rate was $40 \mathrm{Nm}^{3} /$ hour. The solution to be spray-dried was supplied to the pressure nozzle at a $2.5 \mathrm{~mL} /$ minute feed rate using a peristaltic pump. The spray-dried powder was collected from both cyclones using a clean stainless steel microspatula (Sigma-Aldrich).

\section{Spray-dried powder characterization}

Powder flow properties were studied by measuring Hausner's ratio. ${ }^{32}$ An accurately weighed quantity of the powder was carefully transferred to a $10 \mathrm{~mL}$ measuring cylinder. Bulk density was calculated from the initial volume. After 500 taps the volume of powder was noted to be stable and further tapped density was calculated. Hausner's ratio was calculated from the formula (Hausner's ratio = tap density/ bulk density).

Karl Fischer titration (841 Titrando; Metrohm AG, Switzerland) method was employed for determining the moisture content in the particles. Analysis was performed in triplicate and for each analysis $100 \mathrm{mg}$ of powder was used.

Surface morphology and particle size of the spray-dried particles were examined using a Hitachi S-3500N (Tokyo, Japan) scanning electron microscopy (SEM) instrument. The spray-dried particles were sputter coated with gold to make the surface conductive prior to imaging.

$\mathrm{X}$-ray difrraction measurements were conducted on an X'Pert Pro diffractometer (PANalytical Co, Lelyweg, The Netherlands) at a scanning rate of $4 \%$ min in the $2 \theta$ range from $5^{\circ}$ to $40^{\circ}$, with graphite monochromatized $\mathrm{Cu} \mathrm{K \alpha}$ radiation, $5^{\circ} \mathrm{C}-60^{\circ} \mathrm{C}$ in continuous mode.

\section{In vitro evaluation of lung deposition}

An eight-stage cascade impactor (Thermo Fisher Scientific, Waltham, MA, USA) was used for determining fine particle fraction (FPF) and mass median aerodynamic diameter (MMAD). For simulating the human throat, a throat piece was also connected onto the top of the cascade impactor. The accurately weighed spray-dried drug containing SABPEI 5050 particles equivalent to $50 \mathrm{mg}$ of free drug was loaded into the cascade impactor using a Rotahaler ${ }^{\circledR}$ (Cipla, Mumbai, India). Inspiration was done for 1 minute at a $28.3 \mathrm{~L} /$ minute flow rate. After each experiment the particles on each plate were washed with high-performance liquid chromatography (HPLC)-grade methanol and were filled up to $10 \mathrm{~mL}$ using a calibrated standard flask. RIF content of the spray-dried powder was determined by reverse phase HPLC using a Shimadzu Class VP HPLC system (Kyoto, Japan) with a Luna C18 column $(5 \mu \mathrm{m}, 4.6 \mathrm{~mm} \times 250 \mathrm{~mm})$ (Phenomenex, Torrance, CA, USA). Analysis was done in isocratic mode with a mobile phase containing HPLC-grade acetonitrile and water in equal proportion, with an additional $0.03 \%$ TFA. ${ }^{5}$ From the prepared sample $20 \mu \mathrm{L}$ was injected using a Hamilton syringe. Analysis was carried out at a flow rate of $1 \mathrm{~mL} / \mathrm{min}$. The absorbance of the eluant was recorded by ultraviolet (UV) detector at $254 \mathrm{~nm}$. The drug was quantitated using a calibration curve prepared earlier. The cumulative percentage of dose collected from stages 2-5 represents the FPF of the formulation (aerodynamic diameter $1.1-5.8 \mu \mathrm{m})$.

\section{Drug content and drug loading efficiency}

An accurately-weighed quantity $(100 \mathrm{mg})$ of drug-loaded spray-dried particles was lysed using $2 \mathrm{~mL}$ of DCM/MeOH at a 4:1 ratio. The SABPEI 5050 was precipitated by addition of diethyl ether. One milliliter from the supernatant containing RIF was taken in a $10 \mathrm{~mL}$ standard flask and dried under nitrogen flow. The sample was reconstituted in the mobile phase and made up to $10 \mathrm{~mL}$ and analyzed using reverse phase HPLC as explained earlier.

$\%$ Drug content $=\frac{\text { Drug in spray-dried particles } \times 100}{\text { Mass of spray dried particles taken }}$

Drug loading efficiency

$=\frac{\text { Actual drug content in spray-dried particles } \times 100}{\text { Theoretical drug content in spray-dried particles }}$ 


\section{In vitro drug release studies}

The release kinetics study of drug from the spraydried formulation was carried out in PBS ( $\mathrm{pH}$ 7.4). Accurately-weighed microparticles equivalent to $50 \mathrm{mg}$ RIF were dispersed in $5 \mathrm{~mL}$ of the dissolution medium and transferred to pretreated dialysis membrane $(22 \mathrm{~mm} \times 20 \mathrm{~mm}$, molecular weight cut-off 3500 , Serva). The dialysis tubing was then sealed using Teflon clips and was tied to the paddle of a United States Pharmacopeia (USP) type II apparatus (TDT-08 L; Electrolab India, Mumbai, India). The dissolution was done at $37.5 \pm 0.5^{\circ} \mathrm{C}$ with a paddle speed of $150 \mathrm{rpm}$. The volume of dissolution media taken was $1000 \mathrm{~mL}$, and the samples $(5 \mathrm{~mL})$ were taken at $5 \mathrm{~min}$ utes, 15 minutes, 30 minutes, 1 hour, 2 hours, 4 hours, 8 hours, 16 hours, and 24 hours. The samples were taken for analysis in triplicate and the dissolution profile of RIF IP (API) was also generated for comparison. The dissolution samples were analyzed using the HPLC method as explained earlier.

\section{Results and discussion}

Synthesis and physicochemical characterization of SA-grafted BPEI

The hydrophilicity as well as the physical property of BPEI was modified by grafting with SA. SA was covalently conjugated to the primary amino functionality of BPEI in two different weight/weight ratios (5050 and 7030) of SA to BPEI (Scheme 1).

\section{Scheme I}

The covalent conjugation of different SABPEI was confirmed by FTIR and NMR spectroscopy. In addition to the synthesized SABPEI combinations, both BPEI and SA were also analyzed by FTIR (Figure 1). The characteristic aliphatic $-\mathrm{C}-\mathrm{H}$ stretching of the methyl and methylene groups was noticed between $2923-2848 \mathrm{~cm}^{-1}$ for SA (peak 2 and 3 of SA). The presence of primary amino functionality (peak 1 of BPEI) was observed for BPEI. Covalent conjugation was observed in both combinations

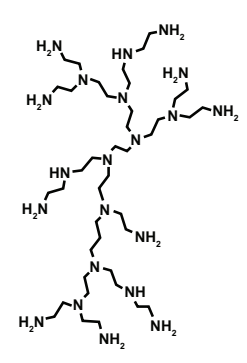

BPEI

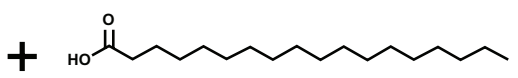

SA
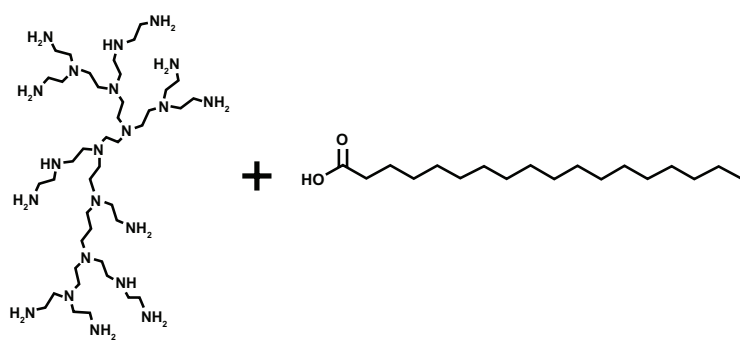

BPEI

SA

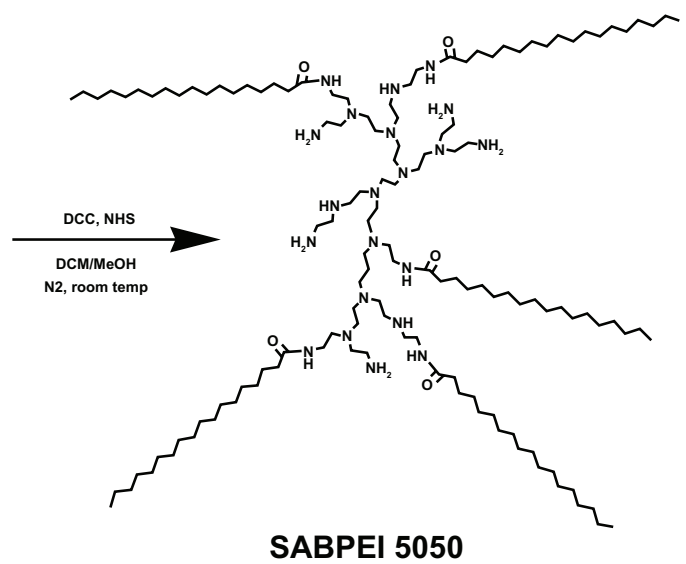

SABPEI 5050

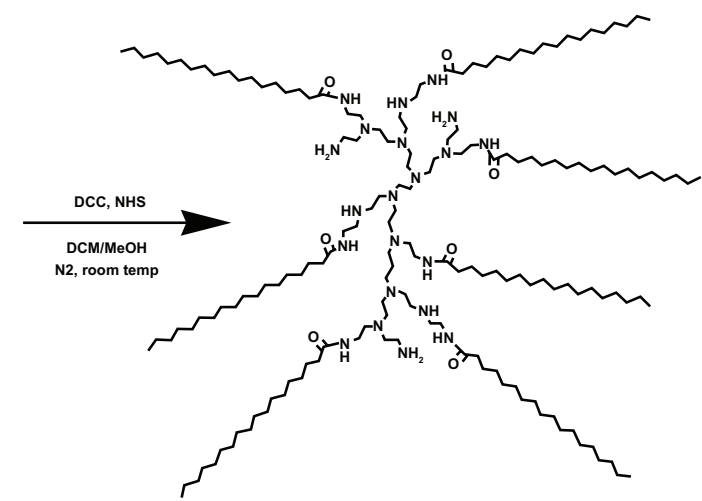

SABPEI 7030

Scheme I Synthesis of SA grafted BPEI with two different combinations.

Abbreviations: SA, stearic acid; BPEI, branched polyethyleneimine; SABPEI 5050, stearic acid-grafted BPEI 5050; SABPEI 7030, stearic acid-grafted BPEI 7030; DCC, N,NDicyclohexylcarbodiimide; NHS, N-hydroxysuccinimide; DCM, Dichloromethane; MeOH, Methanol; N2, Nitrogen gas. 


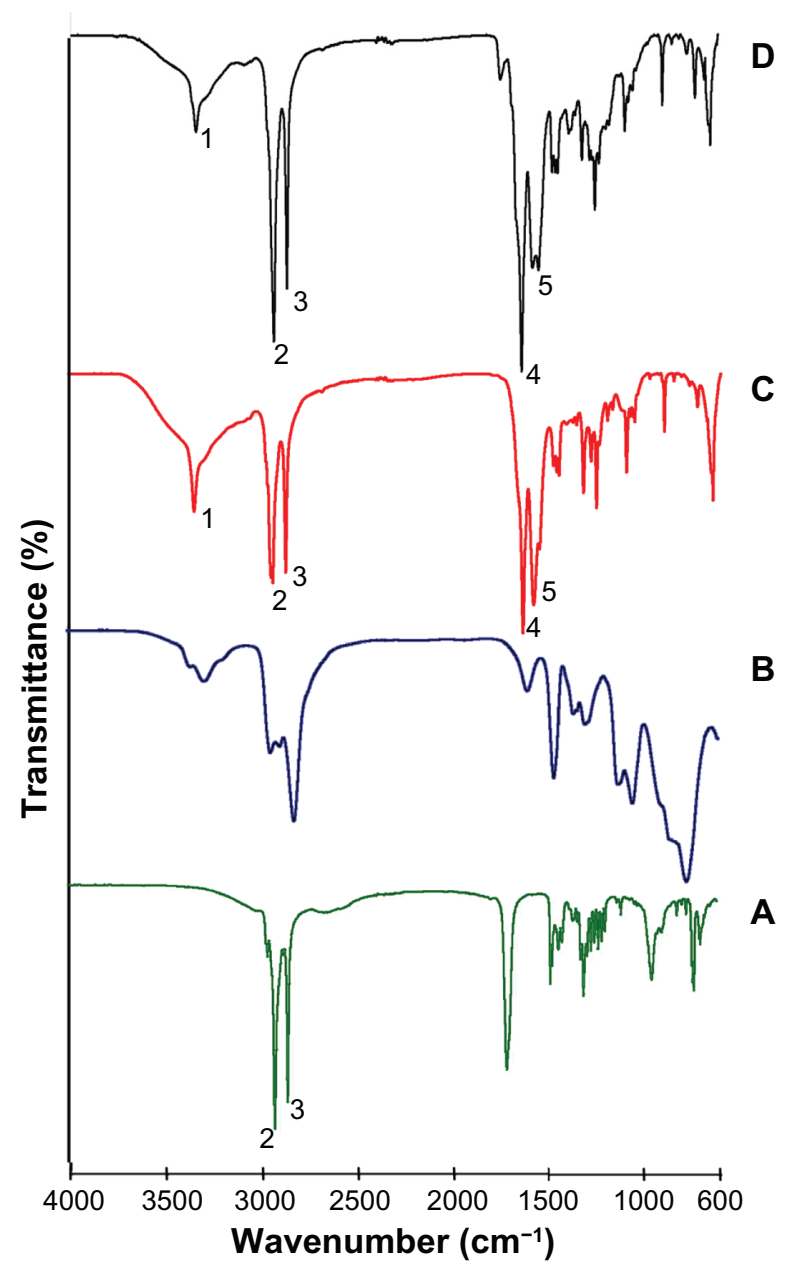

Figure I FTIR spectrum analysis.

Note: FTIR spectrum of (A) SA; (B) BPEl; (C) SABPEI 5050; and (D) SABPEI 7030. Abbreviations: FTIR, Fourier transform infrared; SA, stearic acid; BPEl, branched polyethyleneimine; SABPEI 5050, stearic acid-grafted BPEI 5050; SABPEI 7030, stearic acid-grafted BPEI 7030.

of lipopolymer. A peak corresponding to primary amino group was observed in the range of 3326-3322 cm-1 (Peak 1 of SABPEI 5050 and 7030). The characteristic peaks of an amide linkage was confirmed by the presence of the amide I band in the range of $1626-1625 \mathrm{~cm}^{-1}$ and the amide II band in the range of $1569-1566 \mathrm{~cm}^{-1}$ (peaks 4 and 5). Aliphatic -C-H stretching of the methyl and methylene groups of the SABPEI lipopolymer was observed in the range of $2923-2915 \mathrm{~cm}^{-1}$ and $2850-2848 \mathrm{~cm}^{-1}$ respectively (peaks 2 and 3).

NMR spectroscopic studies were also carried out for structural confirmation of both combinations of SABPEI (Figure 2). Results were as follows: ${ }^{1} \mathrm{H}$ NMR (500 MHz, $\left.\mathrm{CDCl}_{3}, \delta\right), 0.88$ (t, 3H, Peak 1), 1.25 (m, 28H, Peak 2), 1.61-1.67 (m, 2H, Peak 3), 1.91-1.94 (m, 2H, Peak 4), 2.17 (b, 2H, Peak 5), 2.57-2.74 (b, 2H, Peak 6), and 3.46 (m, 2H, Peak 7).

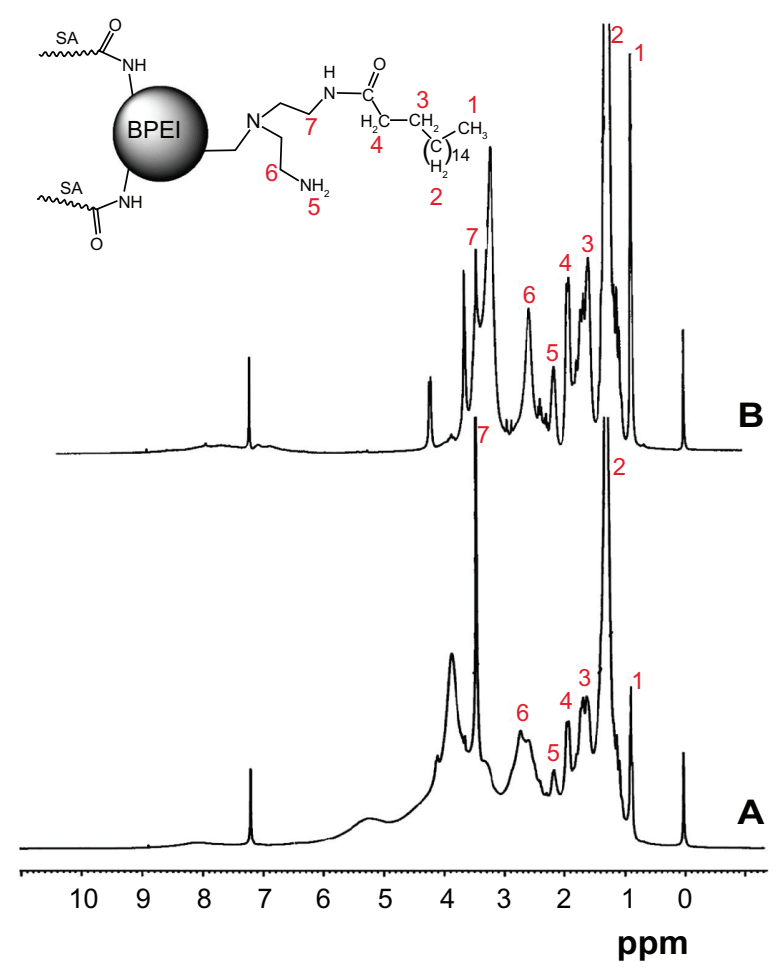

Figure 2 Proton NMR spectrum of SABPEI 5050 and SABPEI 7030. Note: Proton NMR spectrum of (A) SABPEI 5050 and (B) SABPEI 7030. Abbreviations: NMR, nuclear magnetic resonance spectroscopy; BPEl, branched polyethyleneimine; SABPEI 5050, stearic acid-grafted BPEI 5050; SABPEI 7030, stearic acid-grafted BPEI 7030; pPm, parts per million.

\section{Molecular dynamic simulations}

MD studies were conducted in both SABPEI combinations to study the conformational changes that may occur in a polar environment. During simulation of solvation in SPC water for 200 picoseconds (ps) (Figure 3), both combinations behaved differently. At the end of simulation SABPEI 5050's SA moieties were well folded and stabilized to a more stable conformation (Figure 3:1E). Coulombic as well as van der Waals forces of attraction were recorded during the simulation process (Figure 4). It is known that Coulombic and van der Waals forces are opposing forces which play a key role in the size and stability of micelles in polar environments. Van der Waals forces of attraction are reportedly due to the interaction of nonpolar groups, whereas Coulombic forces of repulsion are due to ionization of the functional groups of micelles. ${ }^{33}$ For SABPEI 5050, during the first 90 ps, we observed brief bursts of electrostatic interactions, which were paralleled with steady stabilization of the system by van der Waals interaction. For the rest of the simulation, the system was fairly stable, except for some further stabilization between $140-170$ ps due to electrostatic interactions. Snapshots of SABPEI 7030 in water simulation show that most of the SA moieties were not folded even at the end of 

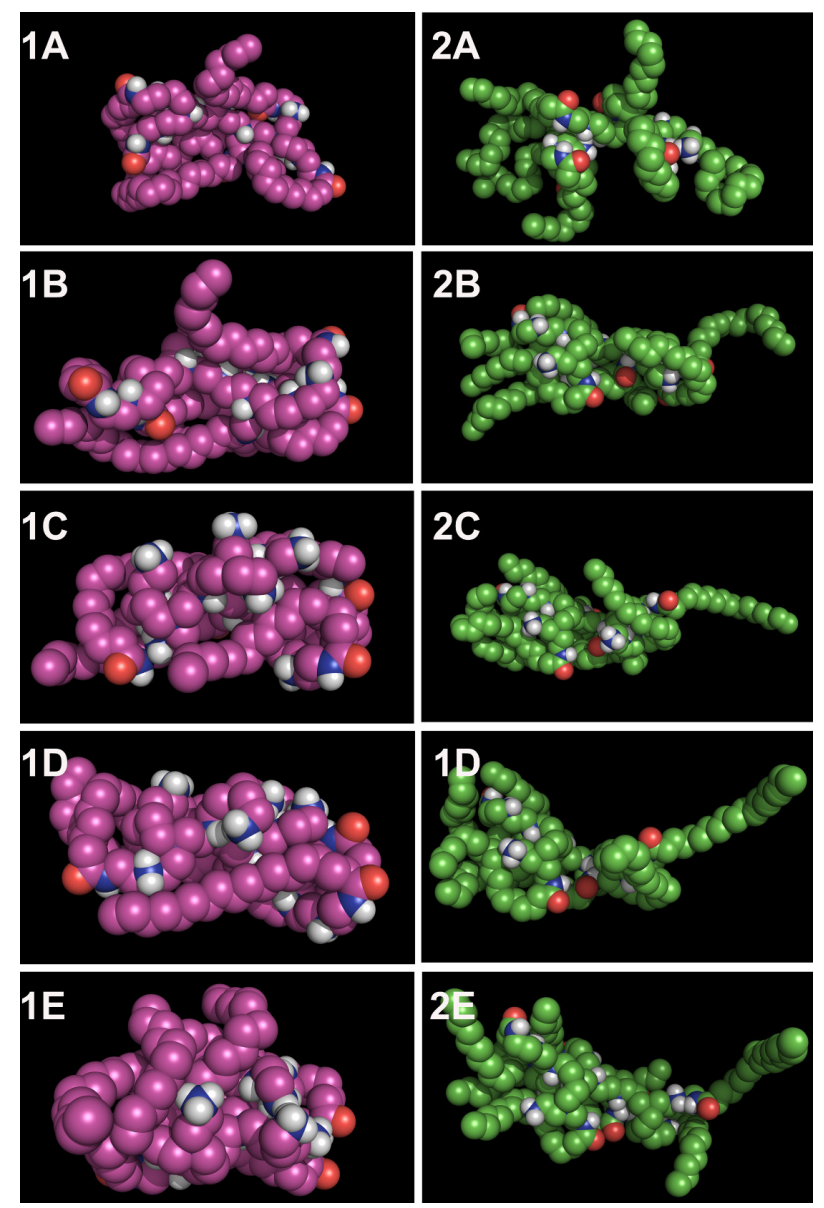

Figure 3 MD simulation folding of SABPEI (5050 and 7030) in aqueous environment.

Note: (IA-E) represent the conformation of SABPEI 5050 at 0, 50, 100, I50, and 200 ps, respectively. (2A-E) represent the conformation of SABPEI 7030 at 0, 50, 100,150 , and $200 \mathrm{ps}$, respectively.

Abbreviations: MD, molecular dynamic; SABPEI 5050, stearic acid-grafted BPEI 5050; SABPEI 7030, stearic acid-grafted BPEI 7030.

$200 \mathrm{ps}$; this resulted in a failure to attain globular topology to the micelles. Coulombic interaction for the SABPEI 7030 combination was found to be increasing after $100 \mathrm{ps}$; interestingly, the van der Waals forces of attraction were stable throughout the simulation. The initial burst of Coulombic forces is expected to be due to the ionization of the free amino groups of SABPEI 5050 into ammonium ions, and further repulsion of like charges. The lower van der Waals forces of attraction in SABPEI 5050 (when compared to SABPEI 7030) are due to the lower proportion of interacting hydrophobic chains (SA). High van der Waals forces in SABPEI 7030, contributed to by a strong nonpolar-nonpolar attraction between SA moieties, lead to decreased flexibility of the micelles. In SABPEI 7030 the SA moieties succeed in effective interaction with adjacent SA and further "pull" of ammonium ions to close proximity. This results in the increased repulsive force (electrostatic interaction) during
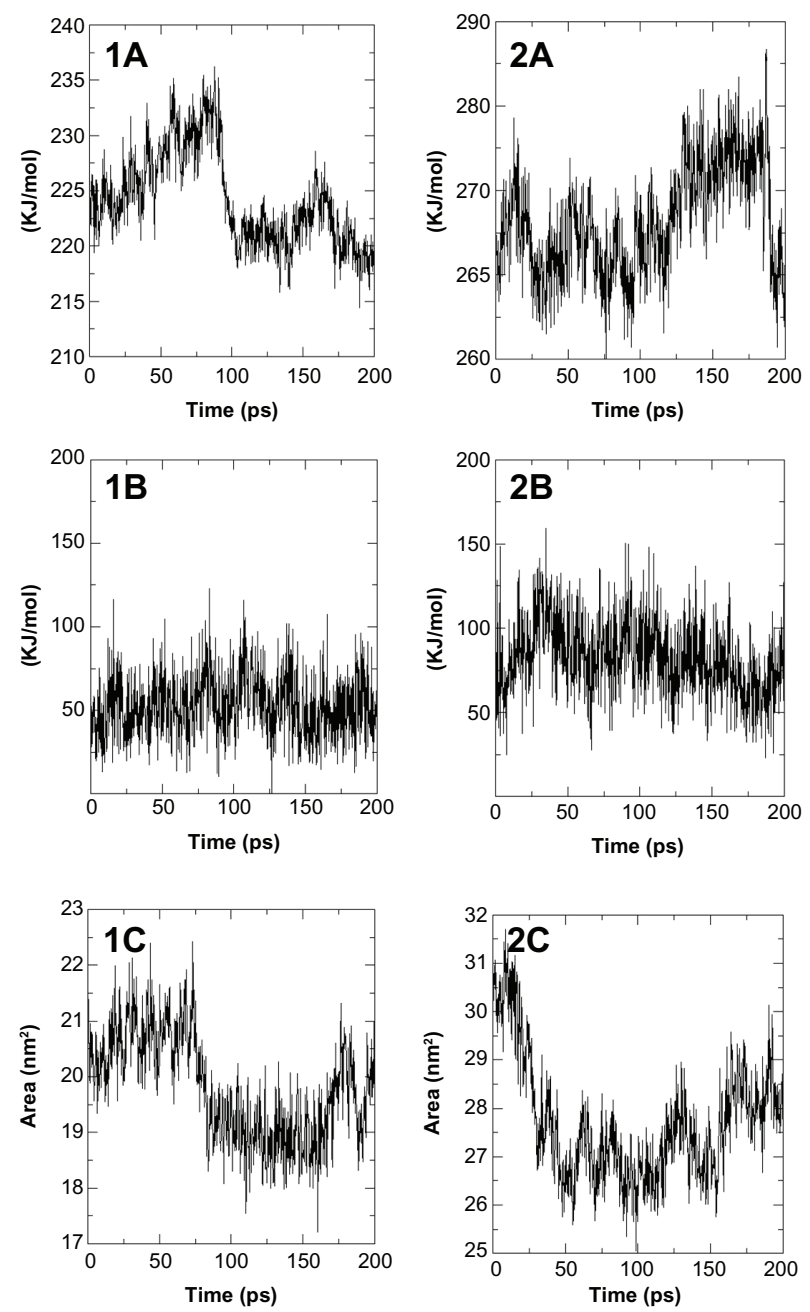

Figure 4 Coulombic interaction, van der Waals forces attraction, and solventaccessible surface area recorded during MD simulation.

Note: (IA-C) represent the changes in Coulombic interaction, van der Waals forces attraction, and solvent accessible surface area for SABPEI 5050. (2A-C) represent the changes in Coulombic interaction, van der Waals forces attraction, and solvent accessible surface area for SABPEI 7030.

Abbreviations: MD, molecular dynamic; SABPEI 5050, stearic acid-grafted BPEI 5050; SABPEI 7030, stearic acid-grafted BPEI 7030.

the simulation. It was observed that stabilization (for both combinations) is not achieved in a single step, but rather the ligand searches for the most stable conformation. The stabilization is strongly achieved by hydrophobic and electrostatic interactions. Van der Waals forces of attraction mainly contribute in deciding the topology of the micelles and micellar growth. Electrostatic repulsive forces save the micelles from aggregation and impart stability in polar environments.

Solvent accessible surface area (SASA) indicated (Figures 4.1C and 4.2C) an amphiphilic property for the SABPEI 5050 combination. In the first $75 \mathrm{ps}$, considerable reduction in surface area was not observed. After attaining stable conformation, due to complete folding of all SA moieties, reduction in SASA was observed. The SABPEI 7030 combination showed 

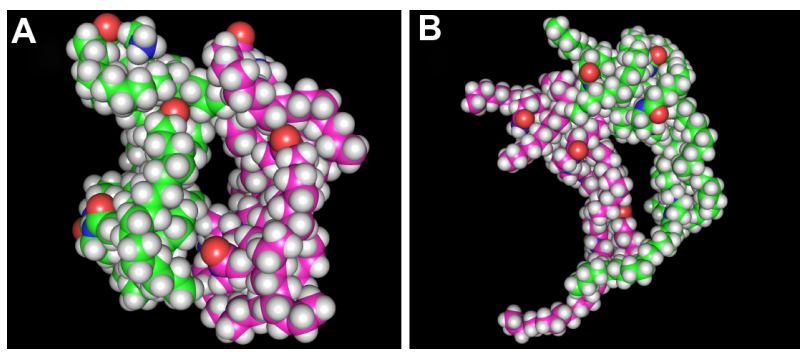

Figure 5 Cohesional forces of attraction between two molecules of micelles in aqueous environment.

Notes: (A) Figure represents SABPEI 5050. (B) Figure represents SABPEI 7030 Abbreviations: SABPEI 5050, stearic acid-grafted BPEI 5050; SABPEI 7030, stearic acid-grafted BPEI 7030.

a sudden decrease in the surface area during simulation. Which is possibly a result of the highly hydrophobic corona of the micelles. Folding of SA moieties in a programmed fashion resulted in the formation of a unimolecular capsule-like coreshell amphiphilic architecture for SABPEI 5050. Similar reverse micelle properties were noted by Stiriba et al on grafting hydrophobic moieties to hyper-branched polyglycerols. ${ }^{34}$ It is reported that unimolecular micelles did not show dynamic equilibrium between individual amphiphiles, which gives superior stability to the micelles. ${ }^{35}$

Intermolecular (cohesional) forces of attraction between the combinations (SABPEI 5050 and 7030) of micelles were also studied through MD simulation (for $500 \mathrm{ps}$ ). In the SABPEI 5050 combination, micelles were observed to be more flexible. Like charges present in the adjacent lipopolymer molecules were found to be aligning in opposite directions to minimize the charge repulsion. In SABPEI
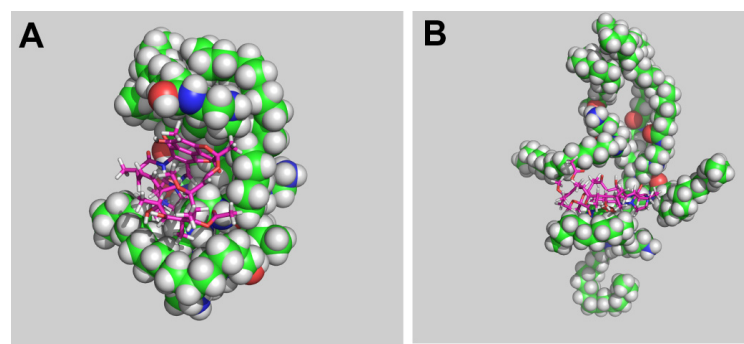

Figure 6 SABPEI micelles folded after docking with rifampicin drug. Notes: (A) SABPEI 5050 micelles folded after docking with rifampicin drug. (B) SABPEI 7030 micelles folded after docking with rifampicin drug.

Abbreviations: SABPEI 5050, stearic acid-grafted BPEI 5050; SABPEI 7030, stearic acid-grafted BPEI 7030.

7030, the lipopolymer's hydrophobic SA moieties were found to interact throughout the simulation. This hampered the plasticity of the molecule and self-alignment of protonated nitrogen (blue spheres in Figure 5) to the opposite direction, as seen in the SABPEI 5050 combination, was not observed.

\section{Drug docking simulation with lipopolymer}

Docking studies of RIF and the SABPEI 5050 combination showed that the drug molecule was well inserted into the micelles (Figure 6). The SABPEI 5050 combination was found to attain a cup-like morphology and to hold the drug molecule in it. In the presence of RIF, folding of stearyl moieties of SABPEI 7030 was more incomplete compared to free SABPEI 7030, which also ascertains the capability of the SABPEI 5050 as an efficient carrier system.

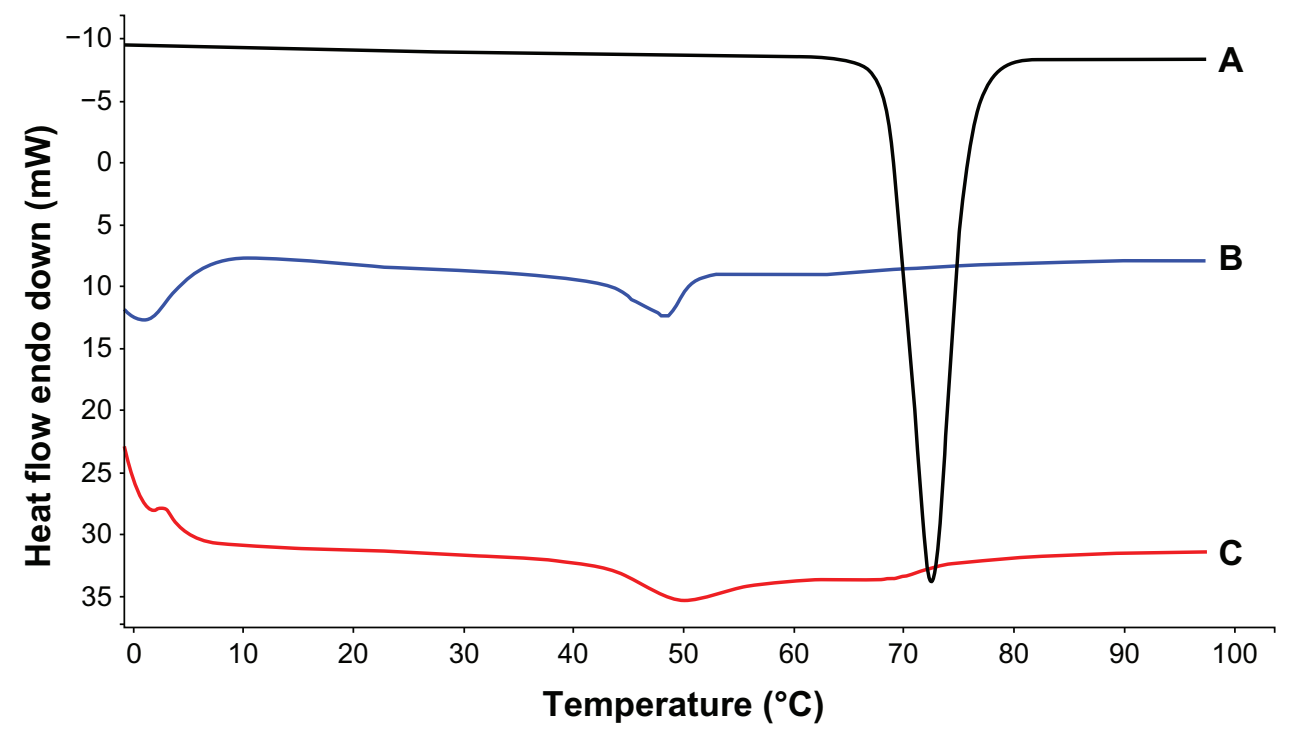

Figure 7 DSC thermogram analysis.

Note: DSC thermograms of (A) stearic acid, (B) SABPEI 5050, and (C) SABPEI 7030.

Abbreviations: DSC, differential scanning calorimetry; SABPEI 5050, stearic acid-grafted BPEI 5050; SABPEI 7030, stearic acid-grafted BPEI 7030. 


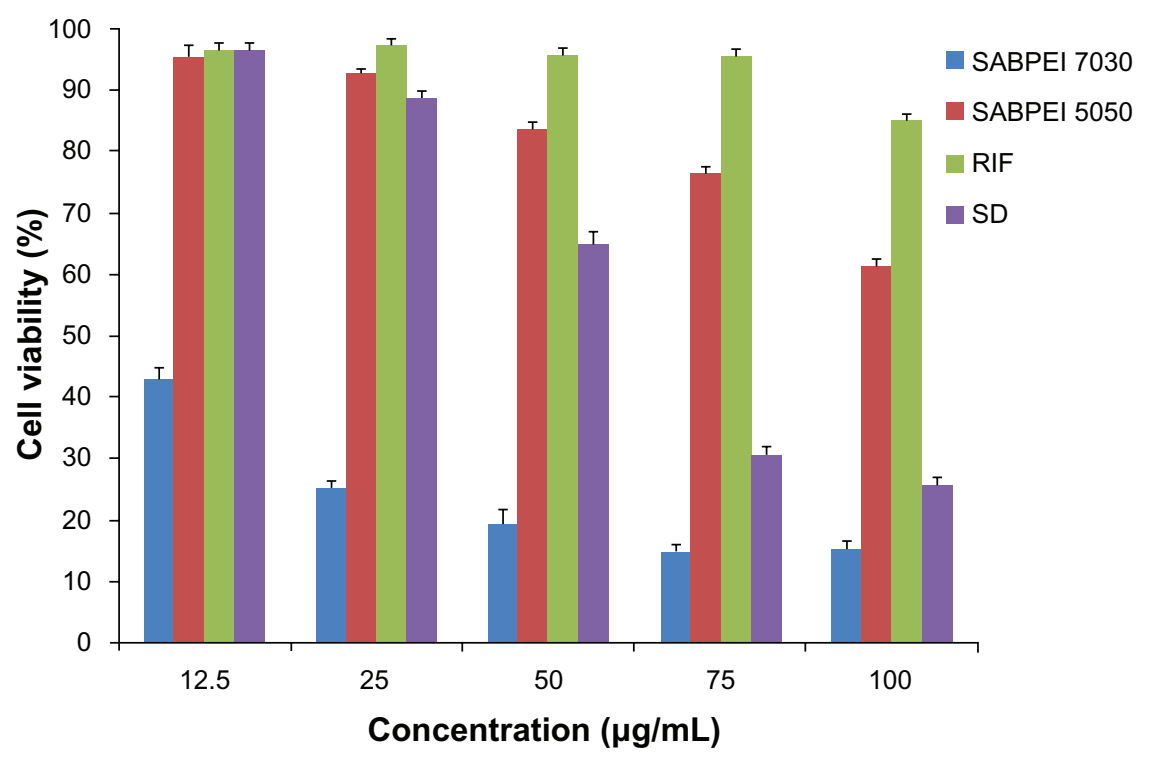

Figure 8 Cell viability of THP-I cells against SABPEI lipopolymers of different combination, rifampicin API, and spray-dried nanomicelles. Abbreviations: THP-I, human acute monocytic leukemia; SABPEI 5050, stearic acid-grafted BPEI 5050; SABPEI 7030, stearic acid-grafted BPEI 7030; API, active pharmaceutical ingredient; RIF, rifampicin; SD, spray-dried nanomicelles.

\section{Physical characterization of lipopolymer}

Differential scanning calorimetric (DSC) studies indicate that the melting point curve of the $\mathrm{SA}\left(\mathrm{Tm} 72.53^{\circ} \mathrm{C}\right)$ was shifted to a lower temperature $\left(\sim 50^{\circ} \mathrm{C}\right)$ among different combinations (Figure 7). This is in accordance with the general observation that crystallization of individual blocks in star shaped copolymer micelles is hampered, resulting in shifting of the endothermic curve. ${ }^{36,37}$ The SABPEI 5050 combination was found to be more crystalline, indicating the ability of folded SA moieties in complete crystallization. Earlier studies on star shaped micelles revealed that micellization ability is directly proportional to crystallinity. As observed in MD simulation studies, the unfolded SA moieties may be succeeding in establishing intermolecular bonds due to the hydrophobic-hydrophobic interaction with the adjacent SABPEI 7030. The presence of both melting and glass transition curves in SABPEI 7030 indicates the existence of a crystalline and amorphous domain. The increased mobility of the unfolded SA in SABPEI 7030 might be creating amorphous domains in the lipopolymer.

On examining the dynamic light scattering (DLS) data, the SABPEI 5050 combination has a smaller size compared to the SABPEI 7030 combination (supporting information S1). The average cumulant particle size (Z-average mean) of SABPEI 5050 was observed to be $218.8 \mathrm{~nm}$, whereas extensive aggregation was observed for SABPEI 7030 (Z-average $1378 \mathrm{~nm}$ ). A higher polydispersity index (PdI, 0.941) was noted for SABPEI 7030. A comparatively more uniform size distribution (PdI of 0.362) of SABPEI 5050 makes the combination a better carrier system compared to SABPEI 7030 (PdI 0.941). In accordance with the simulation study, weak cohesional force of attraction in SABPEI 5050 is inferred to be the major reason for easy dispersion and uniform size distribution of micelles in the aqueous environment. SABPEI 5050 micellar suspension was found to be stable over a period of 1 week, whereas settling of the micelles was observed in the SABPEI 7030 combination. The increased hydrophobicity and cohesional force of attraction are expected to cause larger particle size and aggregation of SABPEI 7030 particles.

Surface charge of the particles was also simultaneously assessed by zeta $(\zeta)$ potential measurement (supporting information S2). The particles of SABPEI 5050 nanomicelles were found to be positively charged $(\zeta=16.7)$. This may be because of the ionization of free amino groups of SABPEI in aqueous medium to ammonium ion $\left(-\mathrm{NH}_{2}\right.$ to $\left.-\mathrm{NH}_{3}^{+}\right)$. A shift in $\zeta$ potential from positive to negative charge $(-10.2)$ was observed in SABPEI 7030. The decrease in the number of ionizable primary amino groups and the introduction of new carbonyl functionality through SA resulted in shifting of cationic property to anionic nature for SABPEI 7030 $(\zeta=-10.2){ }^{38}$

\section{In vitro cytotoxicity and cell uptake study of lipopolymer}

Cytotoxicity of the synthesized SABPEI of different combinations was tested by cell viability assay (Figure 8 ). 

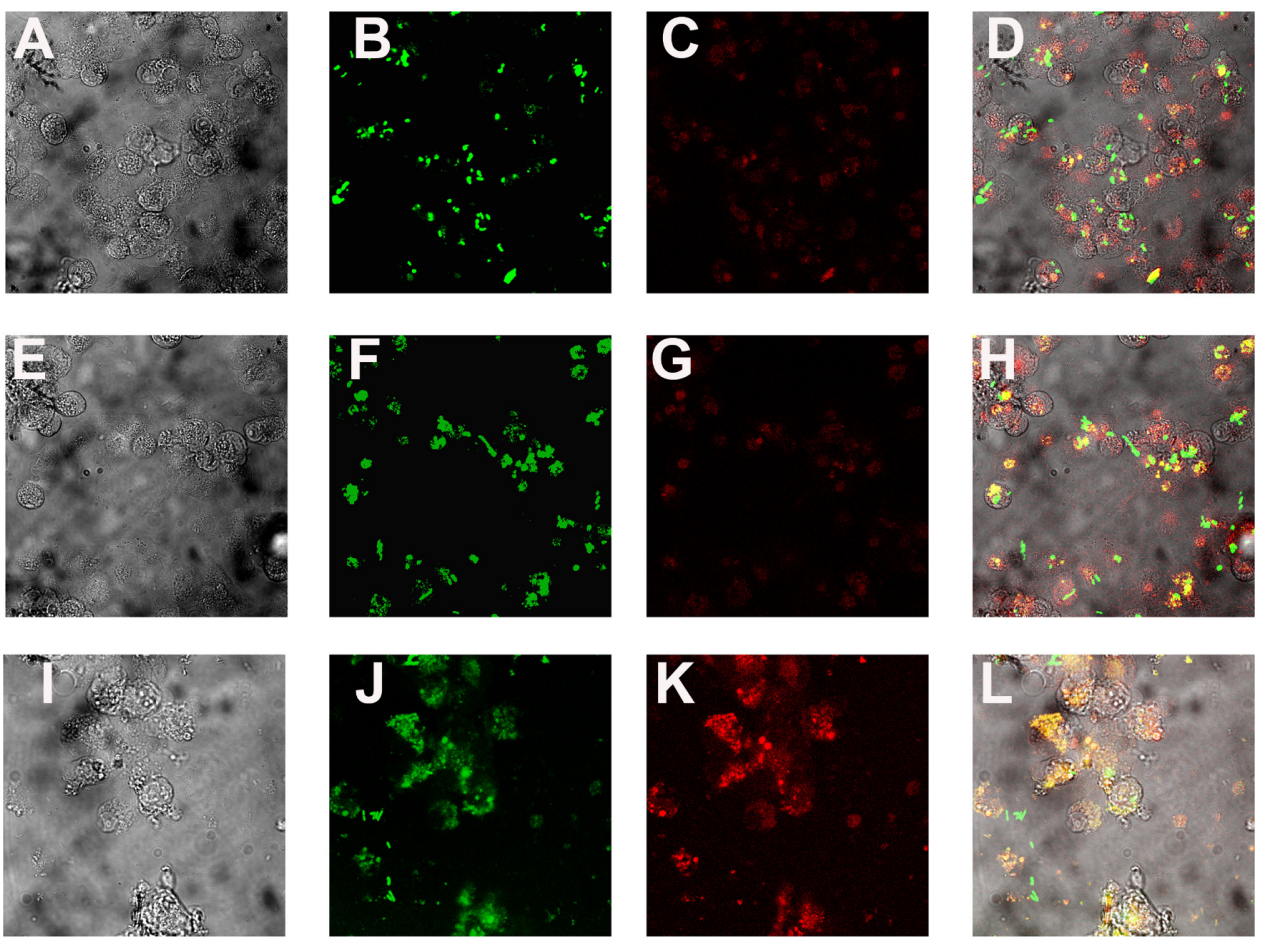

Figure 9 Confocal microscopy images of THP-I macrophage-differentiated cells infected with GFP-tagged Mycobacterium smegmatis after incubating with rhodamineentrapped SABPEI 5050 nanomicelles.

Notes: (A), (E), and (I) are the bright field images of cells fixed after 30 minutes, I hour, and 2 hours incubation, respectively. (B), (F), and (J) are the fluorescent images of GFP-tagged M. smeg after 30 minutes, I hour, and 2 hours, respectively, after incubating with nanomicelles. (C), (G), and (K) are rhodamine-entrapped nanomicelles taken by the cells at 30 minutes, I hour, and 2 hours, respectively. (D), (H), and (L) show colocalization of the nanomicelles and M. smeg inside THP-I cells after 30 minutes, I hour, and 2 hours, respectively.

Abbreviations: THP-I, human acute monocytic leukemia; GFP, green fluorescent protein; SABPEI 5050, stearic acid-grafted BPEI 5050.

Various concentrations $(12.5,25,50,75$, and $100 \mu \mathrm{g} /$ $\mathrm{mL})$ of SABPEI were prepared for all combinations and incubated for 24 hours with THP-1 differentiated to macrophage. The SABPEI 5050 combination was found to be more biocompatible; more than $80 \%$ of cells were viable at the $75 \mu \mathrm{g} / \mathrm{mL}$ concentration. Pure drug did not show any considerable toxicity even at a concentration of $100 \mu \mathrm{g} / \mathrm{mL}$. Spray-dried microparticles containing RIF (SD) were also studied for biocompatibility. Stock solution of SD equivalent to the concentration of lipopolymer was prepared. For SD, cytotoxicity was found to be similar to plain SABPEI 5050 lipopolymer. The more SA-containing SABPEI 7030 showed higher toxicity in all concentrations. This finding is in agreement with the report of Lima et al. They reported saturated fatty acid can cause necrosis in macrophage cells. ${ }^{39}$ Since the cell viability
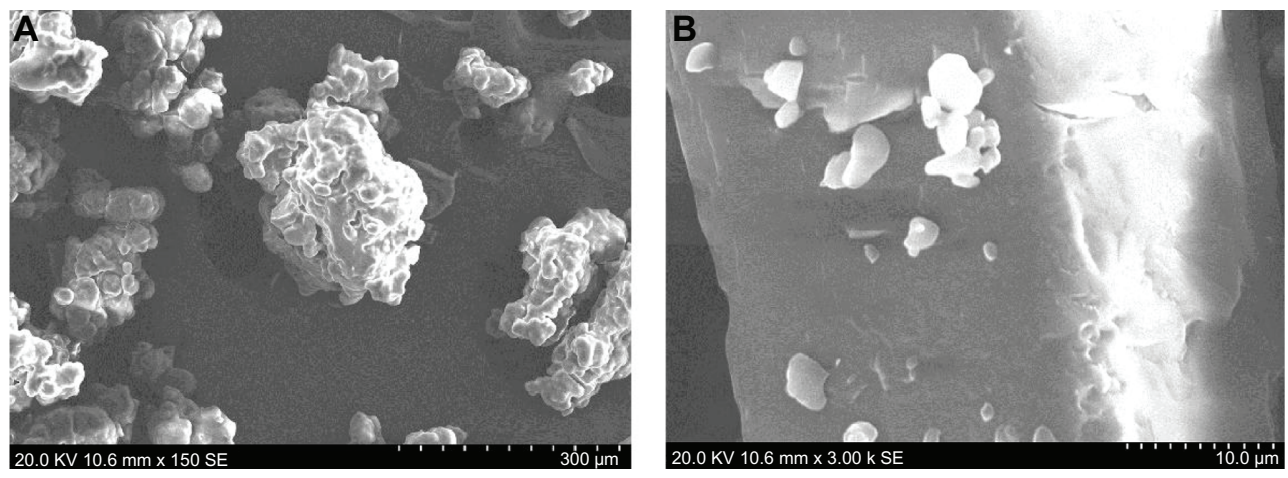

Figure 10 SEM image of SABPEI 5050.

Note: SEM image of SABPEI 5050 cospray-dried with (A) Rifampicin, and (B) spray-dried particles after mixing with Inhalac 230 Meggle Pharma (Hamburg, Germany). Abbreviations: SEM, scanning electron microscopy; BPEI, branched polyethyleneimine; SABPEI 5050, stearic acid-grafted BPEI 5050. 


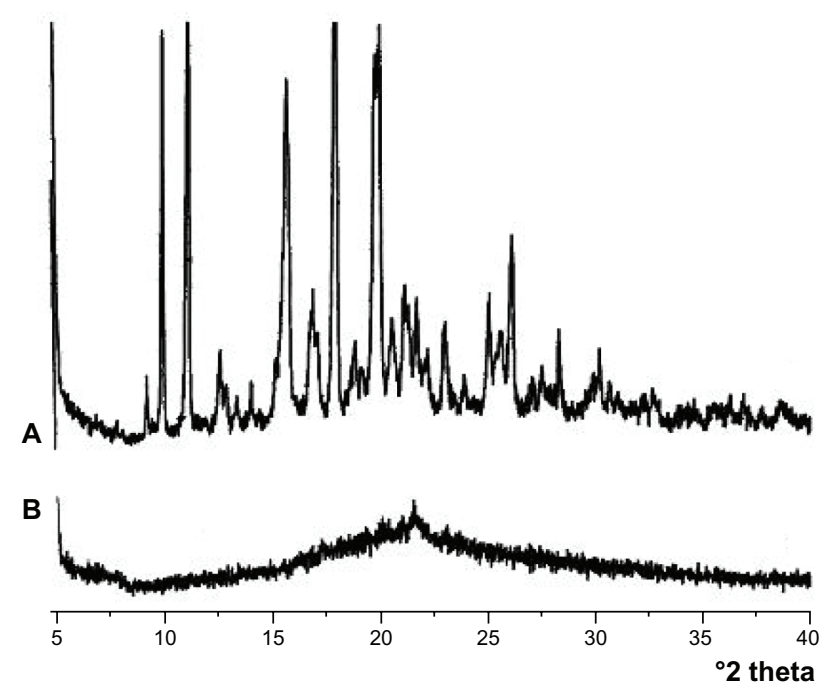

Figure I I PXRD patterns.

Note: PXRD pattern of (A) spray-dried drug loaded nanomicelles and (B) rifampicin API.

Abbreviations: PXRD, powder X-ray diffraction; API, active pharmaceutical ingredient.

assay indicated better biocompatibility of the SABPEI 5050 combination, further studies were conducted.

Cell uptake was studied in macrophage-differentiated THP-1 cell lines after infecting with GFP tagged $M$. smeg. For studying the itinerary of SABPEI 5050 inside the cell, micelles entrapped with rhodamine (a fluorescent dye) were used. Infected cells were incubated with the rhodamine-entrapped SABPEI 5050 combination in the cell culture medium. At different intervals the cells were fixed and imaging was done by confocal microscopy (Figure 9).

Maximum internalization of nanomicelles was found at the end of 2 hours. The smaller particle size and positive $\zeta$ potential may help in absorption of the nanomicelles into the cell through the proton sponging effect. ${ }^{40}$ The first step in cellular uptake for cationic lipid carriers is postulated to be the fusion with the plasma membrane and direct entry into the cytosol. ${ }^{41}$ Instead of perinuclear localization of rhodamine-entrapped nanomicelles as red colored spots, a diffused occurrence of the micelles throughout the cytosol was observed. This indicates a chance of non-endocytics entry into the SABPEI 5050 nanomicelles. ${ }^{42}$ The GFP-tagged green $M$. smeg was found to be localized in specific regions of the cytoplasm. Interestingly, after 1-hour incubation, a larger colocalization of GFP-tagged M. smeg with rhodamineentrapped nanomicelles was observed inside the THP-1 differentiated cell. These positively charged SABPEI 5050 nanomicelles can be employed for targeting Mycobacterium tuberculosis residing inside the phagosome of alveolar macrophage.

\section{Spray-dried powder characterization}

The generated particles were found to have very low bulk density $\left(0.194 \pm 0.013 \mathrm{~g} / \mathrm{cm}^{3}\right)$. Fast evaporation of the low boiling point solvents used for the spray-drying process

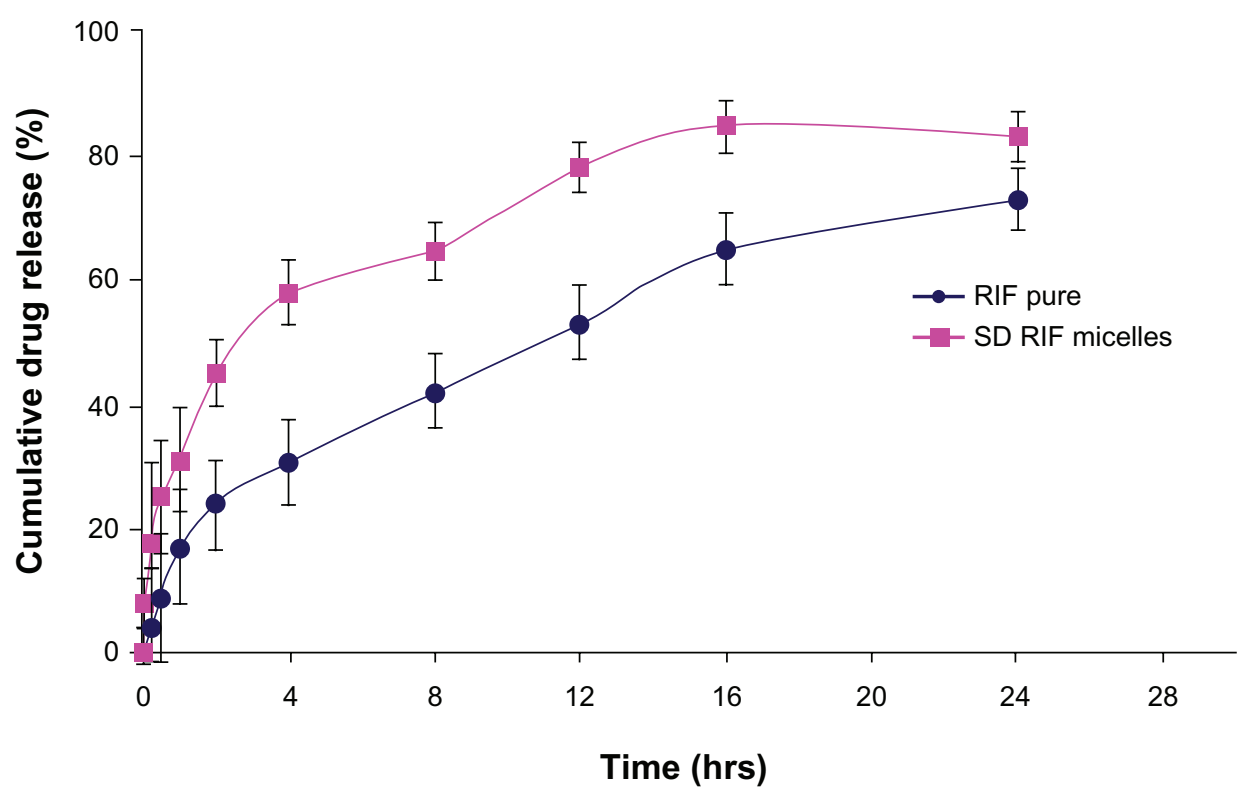

Figure 12 Drug release profile of rifampicin from spray-dried SABPEI 5050 nanomicelles in comparison with pure rifampicin.

Abbreviations: SD RIF micelles, spray-dried SABPEI 5050 nanomicelles; RIF pure, pure rifampicin; hrs, hours; SABPEI 5050, stearic acid-grafted BPEI 5050. 
might have helped in formation of hollow or porous particles, resulting in low bulk density. According to United States Pharmacopeia (USP) classification (Chapter 1174, powder flow) Hausner's ratio $1.21 \pm 0.014$ indicates the particles have "fair" flow properties.

Moisture content plays a crucial role in stability and particle aggregation on storage. It was observed that use of organic solvents and compressed nitrogen gas used for aerolization helped in maintaining low (1.54\%) moisture content in the formulation.

Topological studies (Figure 10) were carried out using SEM. The SEM images showed the spray-dried particles in aggregated form. Lactose is a US FDA approved excipient commonly used in DPI formulations for adsorbing drug particles. The RIF-loaded spray-dried SABPEI particles were blended with inhalation grade lactose (Inhalac 230; Meggle Pharma) and SEM imaging was done. Interestingly, spherical morphology was observed for the adsorbed drug-loaded particles and the population of particles were more on the asperities of the lactose crystals. It is reported that asperities of the lactose crystal act as "active sites". The particles were found to be under the inhalable size range (5 microns).

Powder X-ray diffraction analysis (Figure 11) revealed that the RIF-loaded SABPEI 5050 existed in the amorphous state. Interestingly, both drug and carrier were transformed from a crystalline to an amorphous state during the process. Since the polarity of the spray-drying solution (DCM/ $\mathrm{MeOH}$ ) is very low, the SABPEI 5050 lipopolymer is possibly in an unfolded state in the particle. It was also found that RIF exists in a molecular dispersion state in the spray-dried particle. When lipopolymer is in the amorphous condition, the drug loading efficiency is improved, but when SD drugloaded SABPEI 5050 is exposed to a polar environment (eg, lung fluid), folding of SA moieties and further crystallization of SA is expected. A crystalline state is desirable for micellar stability and sustained drug release. ${ }^{43}$

\section{Determination of in vitro lung deposition efficiency}

Considerable difference in MMAD was observed for free drug $(4.86-5.21 \mu \mathrm{m})$ and for drug loaded SABPEI 5050 $(2.31-2.45 \mu \mathrm{m})$. This indicates amorphization of API in SABPEI 5050 lipopolymer matrix and the existence of hollow microparticles of respirable size range after the spray-drying process. The FPF data show that most of the SABPEI 5050 spray-dried drug-loaded particles come in the inhalable size range $(67.88 \% \pm 3 \%)$.

\section{Drug loading and in vitro release kinetics}

A reverse phase HPLC technique was used for the determination of drug loading and quantification of RIF in the dissolution medium samples. Drug content was determined to be $48 \%$ of dry weight of the spray-dried powder. About $99 \%$ drug loading was observed in spray-dried drug-loaded SABPEI 5050 micelles. In vitro release kinetics were done in PBS (pH 7.4) to simulate the lung fluid $\mathrm{pH}$ condition.

The drug release profile (Figure 12) from spray-dried particles was compared with free drug. Initial drug release from the spray-dried formulation was high (burst release), compared to the RIF API. It is reported that solubility of RIF in neutral $\mathrm{pH}$ is very low $(1.4 \mathrm{mg} / \mathrm{mL})$. Since the formulation contains about $50 \%$ of the drug, a larger portion of the drug may be in unentrapped state or merely adsorbed to the lipopolymer. The amorphization of RIF API during spray drying will also result in the solubility enhancement and further burst release of drug. After 2 hours, controlled release of the drug was observed. This may be resultant from holding of the drug in the SABPEI 5050 micelles by electrostatic forces, as observed in computational docking studies. The conversion of SABPEI 5050 from amorphous state to crystalline micellar state in PBS is also expected to be one of the reasons for the controlled delivery of drug over a period of 24 hours. Only $73 \%$ of the drug was released from free RIF API, whereas about $85 \%$ of drug was released from the SABPEI 5050 micelles in a period of 24 hours. The initial burst release of RIF may help in achieving the loading dose at a greater pace and further controlled release may help in maintaining the concentration of drug in the target.

\section{Conclusions}

In summary, we have synthesized SA-grafted branched polyethylenimine. It was found that long alkyl moiety of SA plays an important role in the self-assembling nature of the micelles. An increase in SA proportion imparts less water dispersibility and also shifts zeta potential from a positive value to a negative value. Even though SA is an endogenous fatty acid, the SABPEI 5050 combination was found to be the more biocompatible lipopolymer when compared to SABPEI 7030. This clearly indicates the role of particle size and surface charge on cytotoxicity. The cationic property of the SABPEI 5050 combination's nanomicelles was found to affect the uptake into the cell due to the proton sponging effect. Colocalization of rhodamine-entrapped nanomicelles with $M$. smeg indicates the prospective use of these nanomicelles for targeting various cell compartments of alveolar macrophages. The spray-dried drug-loaded nanomicelles also proved to be a better excipient for delivering xenobiotics to lung through dry powder inhalation 
technology. This study ascertains the importance of a computational approach in carrier designing, drug-carrier interactions, and stability of carrier in experimental conditions.

\section{Acknowledgments}

MVV is thankful to ICMR for providing senior research fellowship. Authors are thankful to RP Deshmukh of labultima for providing spray-drying facility. We are grateful to Department of Biotechnology, India and Rajiv Gandhi Centre for Biotechnology for providing the research facility. P. Guruswamy, NIIST Thiruvananthapuram, for PXRD analysis.

\section{Disclosure}

The authors report no conflicts of interest in this work.

\section{References}

1. Muttil P, Kaur J, Kumar K, Yadav AB, Sharma R, Misra A. Inhalable microparticles containing large payload of anti-tuberculosis drugs. Eur J Pharm Sci. 2007;32(2):140-150.

2. Cochrane MG, Bala MV, Downs KE, Mauskopf J, Ben-Joseph RH. Inhaled corticosteroids for asthma therapy: patient compliance, devices, and inhalation technique. Chest. 2000;117(2):542-550.

3. Pilcer G, Amighi K. Formulation strategy and use of excipients in pulmonary drug delivery. Int J Pharm. 2010;392(1-2):1-19.

4. Cook RO, Pannu RK, Kellaway IW. Novel sustained release microspheres for pulmonary drug delivery. J Control Release. 2005;104(1):79-90.

5. Coowanitwong I, Arya V, Kulvanich P, Hochhaus G. Slow release formulations of inhaled rifampin. AAPS J. 2008;10(2):342-348.

6. Esmaeili F, Hosseini-Nasr M, Rad-Malekshahi M, Samadi N, Atyabi F, Dinarvand R. Preparation and antibacterial activity evaluation of rifampicin-loaded poly lactide-co-glycolide nanoparticles Nanomedicine. 2007;3(2):161-167.

7. Tsapis N, Bennett D, O'Driscoll K, et al. Direct lung delivery of para-aminosalicylic acid by aerosol particles. Tuberculosis (Edinb). 2003;83(6):379-385.

8. Suarez S, O'Hara P, Kazantseva M, et al. Respirable PLGA microspheres containing rifampicin for the treatment of tuberculosis: screening in an infectious disease model. Pharmaceutical research. 2001;18(9):1315-1319.

9. O’Hara P, Hickey AJ. Respirable PLGA microspheres containing rifampicin for the treatment of tuberculosis: manufacture and characterization. Pharm Res. 2000;17(8):955-961.

10. Suarez S, O'Hara P, Kazantseva M, et al. Respirable PLGA microspheres containing rifampicin for the treatment of tuberculosis: screening in an infectious disease model. Pharm Res. 2001;18(9): 1315-1319.

11. De S, Robinson DH. Particle size and temperature effect on the physical stability of PLGA nanospheres and microspheres containing Bodipy. AAPS Pharm Sci Tech. 2004;5(4):e53.

12. Nair KL, Jagadeeshan S, Nair SA, Kumar GS. Biological evaluation of 5-fluorouracil nanoparticles for cancer chemotherapy and its dependence on the carrier, PLGA. Int J Nanomed. 2011;6: 1685-1697.

13. Ashwanikumar N, Kumar NA, Nair SA, Kumar GS. Methacrylic-based nanogels for the $\mathrm{pH}$-sensitive delivery of 5-fluorouracil in the colon. Int J of Nanomed. 2012;7:5769-5779.

14. Cho H, Kwon GS. Polymeric micelles for neoadjuvant cancer therapy and tumor-primed optical imaging. ACS Nano. 2011;5(11): 8721-8729.
15. Du YZ, Weng Q, Yuan H, Hu FQ. Synthesis and antitumor activity of stearate-g-dextran micelles for intracellular doxorubicin delivery. ACS Nano. 2010;4(11):6894-6902.

16. Gu F, Zhang L, Teply BA, et al. Precise engineering of targeted nanoparticles by using self-assembled biointegrated block copolymers. Proc Natl Acad Sci U S A. 2008;105(7):2586-2591.

17. Hans M, Shimoni K, Danino D, Siegel SJ, Lowman A. Synthesis and characterization of mpeg-PLA prodrug micelles. Biomacromolecules. 2005;6(5):2708-2717.

18. Huynh L, Neale C, Pomès R, Allen C. Computational approaches to the rational design of nanoemulsions, polymeric micelles, and dendrimers for drug delivery. Nanomedicine: Nanotechnology, Biology and Medicine. 2012;8(1):20-36.

19. Samanta $S$, Roccatano D. Interaction of curcumin with PEO-PPO-PEO triblock copolymers: a molecular dynamics study. $J$ Phys Chem $B$. 2013;117(11):3250-3257.

20. Dehshahri A, Oskuee RK, Shier WT, Hatefi A, Ramezani M. Gene transfer efficiency of high primary amine content, hydrophobic, alkyloligoamine derivatives of polyethylenimine. Biomaterials. 2009;30(25): 4187-4194.

21. Wen Y, Pan S, Luo X, Zhang X, Zhang W, Feng M. A biodegradable low molecular weight polyethylenimine derivative as low toxicity and efficient gene vector. Bioconjug Chem. 2009;20(2):322-332.

22. Bivas-Benita M, Romeijn S, Junginger HE, Borchard G. PLGA-PEI nanoparticles for gene delivery to pulmonary epithelium. Eur J Pharm Biopharm. 2004;58(1):1-6.

23. Lindahl E, Hess B, Van Der Spoel D. GROMACS 3.0: a package for molecular simulation and trajectory analysis. J Mol Model. 2001;7(8): 306-317.

24. van Aalten DM, Bywater R, Findlay JB, Hendlich M, Hooft RW, Vriend G. PRODRG, a program for generating molecular topologies and unique molecular descriptors from coordinates of small molecules. J Comput Aided Mol Des. 1996;10(3):255-262.

25. Berendsen HJC, Postma JPM, van Gunsteren WF, Hermans J. Interaction models for water in relation to protein hydration. In: Pullman B, editor. Intermolecular Forces. Reidel Publishing Company: Dordrecht, The Netherlands; 1981:331-342.

26. Hess B, Bekker H, Berendsen HJC, Fraaije JGE. M. LINCS: a linear constraint solver for molecular simulations. J Comput Chem. 1997;18(12):1463-1472.

27. Miyamoto S, Kollman PA. SETTLE: an analytical version of the SHAKE and RATTLE algorithm for rigid water models. $J$ Comput Chem. 1992;13(8):952-962.

28. Darden T, York D, Pedersen L. Particle mesh Ewald: an N $\log (\mathrm{N})$ method for Ewald sums in large systems. J Chem Phys. 1993;98(12): 10089-10092.

29. Berendsen HJC, Postma JPM, van Gunsteren WF, DiNola A, Haak JR. Molecular dynamics with coupling to an external bath. J Chem Phys. 1984;81(8):3684-3691.

30. Riendeau CJ, Kornfeld H. THP-1 cell apoptosis in response to mycobacterial infection. Infect Immun. 2003;71(1):254-259.

31. Lecároz C, Blanco-Prieto MJ, Burrell MA, Gamazo C. Intracellular killing of Brucella melitensis in human macrophages with microsphere-encapsulated gentamicin. $J$ Antimicrob Chemother. 2006;58(3):549-556.

32. Hamishehkar H, Emami J, Najafabadi AR, et al. Effect of carrier morphology and surface characteristics on the development of respirable PLGA microcapsules for sustained-release pulmonary delivery of insulin. Int J Pharm. 2010;389(1):74-85.

33. Lazaridis T, Mallik B, Chen Y. Implicit solvent simulations of DPC micelle formation. J Phys Chem B. 2005;109(31):15098-15106.

34. Stiriba SE, Kautz H, Frey H. Hyperbranched molecular nanocapsules: comparison of the hyperbranched architecture with the perfect linear analogue. J Am Chem Soc. 2002;124(33):9698-9699.

35. Meier MAR, Gohy JF, Fustin CA, Schubert US. Combinatorial synthesis of star-shaped block copolymers: host-guest chemistry of unimolecular reversed micelles. J Am Chem Soc. 2004;126(37):11517-11521. 
36. Hyun H, Cho JS, Kim BS, et al. Comparison of micelles formed by amphiphilic star block copolymers prepared in the presence of a nonmetallic monomer activator. J Polym Sci Pol Chem. 2008;46(6): 2084-2096.

37. Moretton MA, Glisoni RJ, Chiappetta DA, Sosnik A. Molecular implications in the nanoencapsulation of the anti-tuberculosis drug rifampicin within flower-like polymeric micelles. Colloids Surf B Biointerfaces. 2010;79(2):467-479.

38. Zhang Q, Yie G, Li Y, Yang Q, Nagai T. Studies on the cyclosporin A loaded stearic acid nanoparticles. Int J Pharm. 2000;200(2):153-159.

39. Martins de Lima T, Cury-Boaventura M, Giannocco G, Nunes M, Curi R. Comparative toxicity of fatty acids on a macrophage cell line (J774). Clinical Science. 2006;111:307-317.
40. Yezhelyev MV, Qi L, O’Regan RM, Nie S, Gao X. Proton-sponge coated quantum dots for sirna delivery and intracellular imaging. $\mathrm{J} \mathrm{Am} \mathrm{Chem}$ Soc. 2008;130(28):9006-9012.

41. Khalil IA, Kogure K, Akita H, Harashima H. Uptake pathways and subsequent intracellular trafficking in nonviral gene delivery. Pharmacol Rev. 2006;58(1):32-45.

42. Zhang X, Sharma KK, Boeglin M, et al. Transfection ability and intracellular DNA pathway of nanostructured gene-delivery systems. Nano Lett. 2008;8(8):2432-2436.

43. Yan J, Ye Z, Chen M, et al. Fine tuning micellar core-forming block of poly(ethylene glycol)-block-poly( $\varepsilon$-caprolactone) amphiphilic copolymers based on chemical modification for the solubilization and delivery of doxorubicin. Biomacromolecules. 2011;12(7):2562-2572. 


\section{Supplementary figures}

A

$\begin{array}{rllll} & & \text { Size (d.nm): } & \text { \% intensity } & \text { Width (d.nm): } \\ \text { Z-average (d.nm): } 218.8 & \text { Peak 1: } 257.9 & 97.7 & 141.9 \\ \text { Pdl: } 0.362 & \text { Peak 2: } 5365 & 2.3 & 332.7 \\ \text { Intercept: 0.816 } & \text { Peak 3: } 0.000 & 0.0 & 0.000 \\ \text { Result quality: good } & & & & \end{array}$

Size distribution by intensity

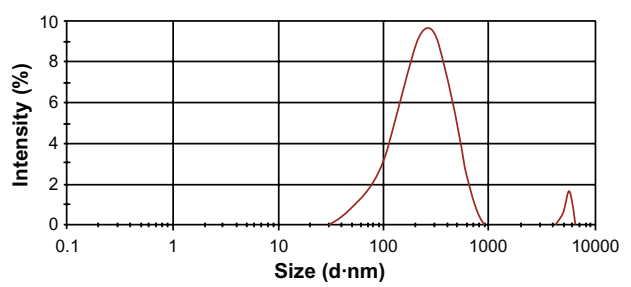

B

$\begin{array}{rrlll}\text { B } & & \text { Size (d.nm): } & \text { \% intensity } & \text { Width (d.nm): } \\ \text { Z-average (d.nm): } 1378 & \text { Peak 1: } & 249.5 & 100.0 & 23.46 \\ \text { Pdl: } 0.941 & \text { Peak 2: } & 0.000 & 0.0 & 0.000 \\ \text { Intercept: } 0.750 & \text { Peak 3: } & 0.000 & 0.0 & 0.000\end{array}$

Size distribution by intensity

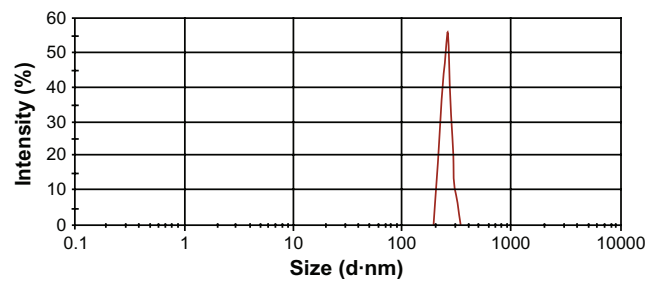

Figure SI Particle size distribution of SABPEI combinations in water.

Note: Particle size distribution of (A) SABPEI 5050 and (B) SABPEl 7030 in water.

Abbreviations: SA, stearic acid; BPEI, branched polyethyleneimine; SABPEI 5050, stearic acid-grafted BPEI 5050; SABPEI 7030, stearic acid-grafted BPEI 7030; Pdl, polydispersity index.

A Zeta potential $(\mathrm{mV}): 16.7$
Zeta deviation $(\mathrm{mV}): 6.92$
Conductivity $(\mathrm{mS} / \mathrm{cm}): 0.0832$ Conductivity $(\mathrm{mS} / \mathrm{cm}): 0.0832$
Result quality: good

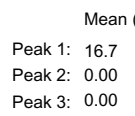

Mean $(\mathrm{mV})$

Area $(\%) \quad$ Width $(\mathrm{mV})$

$\begin{array}{ll}100.0 & 6.92\end{array}$

Peak 3: 0.00

0.0

0.0

Zeta potential distribution

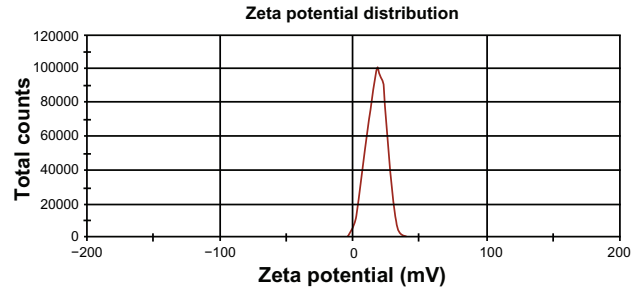

B \begin{tabular}{llll} 
Zeta potential $(\mathrm{mV}):-10.2$ & \multicolumn{1}{c}{ Mean $(\mathrm{mV})$} & Area $(\%)$ & Width \\
Zeta deviation $(\mathrm{mV}): 4.72$ & Peak 1: -10.2 & 100.0 & 4.72 \\
Coeak 2: 0.00 & 0.0 & 0.00
\end{tabular} Conductivity (mS/cm): 0.194 Result quality: good

Peak 3: 0.00

0.0

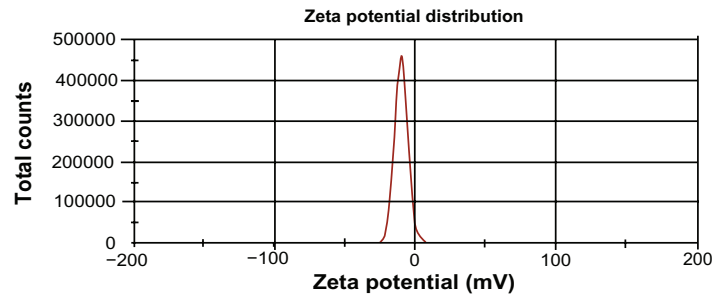

Figure S2 Zeta potential curve of SABPEl combinations.

Note: Zeta potential curve of (A) SABPEI 5050 and (B) SABPEI 7030.

Abbreviations: SA, stearic acid; BPEI, branched polyethyleneimine; SABPEI 5050, stearic acid-grafted BPEI 5050; SABPEI 7030, stearic acid-grafted BPEI 7030; Pdl, polydispersity index.

International Journal of Nanomedicine

\section{Publish your work in this journal}

The International Journal of Nanomedicine is an international, peerreviewed journal focusing on the application of nanotechnology in diagnostics, therapeutics, and drug delivery systems throughout the biomedical field. This journal is indexed on PubMed Central, MedLine, CAS, SciSearch ${ }^{\circledR}$, Current Contents ${ }^{\circledR} /$ Clinical Medicine,

\section{Dovepress}

Journal Citation Reports/Science Edition, EMBase, Scopus and the Elsevier Bibliographic databases. The manuscript management system is completely online and includes a very quick and fair peer-review system, which is all easy to use. Visit http://www.dovepress.com/ testimonials.php to read real quotes from published authors. 